\title{
EHMTI-0262. Dysregulation of inflammatory pathways in a familial hemiplegic migraine 1 mouse model after the induction of cortical spreading depression
}

\author{
B de Vries ${ }^{1 *}$, EE Eising ${ }^{1}$, R Shyti ${ }^{1}$, LS Vijfhuizen ${ }^{1}$, LAM Broos ${ }^{1}$, PAC 't Hoen ${ }^{1}$, MD Ferrari ${ }^{2}$, EA Tolner ${ }^{1}$, \\ AMJM van den Maagdenberg ${ }^{1}$
}

From 4th European Headache and Migraine Trust International Congress: EHMTIC 2014

Copenhagen, Denmark. 18-21 September 2014

\section{Background}

Familial Hemiplegic Migraine type 1 (FHM1) is a rare monogenic subtype of migraine with aura caused by mutations in the CACNA1A gene. In FHM1 knock-in mouse models these mutations increase the susceptibility for cortical spreading depression (CSD): the underlying mechanism of the migraine aura.

\section{Aim}

To study the consequences of CSD in a migraine-relevant context, we measured cortical gene expression profiles in FHM1 and wild-type mice 24 hours after CSD induction.

\section{Method}

Expression profiles were generated using deep-Serial Analysis of Gene Expression (SAGE) sequencing, a tagbased next-generation sequencing method for gene expression profiling. Relevant expression changes were validated by qPCR experiments.

\section{Results}

Our data show that CSD induces differential expression of genes involved in inflammatory pathways in both the FHM1 and wild-type mice. However, we identified a gene set that is up-regulated upon CSD specifically in the FHM1 migraine mouse model. Genes from this gene set are involved in inflammatory and interferon-related signaling, and were often found up-regulated in immunestimulated conditions.

\section{Conclusion}

Differential expression of genes involved in inflammatory pathways in the brain of FHM1 migraine mice compared to wild-type mice upon CSD, indicates that CSD affects the brain differently in a genetically predisposed animal which may help increase our understanding of migraine pathophysiology.

No conflict of interest

\section{Authors' details}

'Human Genetics, Leiden University Medical Center (LUMC), Leiden, Netherlands. ${ }^{2}$ Neurology, Leiden University Medical Center (LUMC), Leiden, Netherlands.

\section{Published: 18 September 2014}

doi:10.1186/1129-2377-15-S1-B5

Cite this article as: de Vries et al: EHMTI-0262. Dysregulation of inflammatory pathways in a familial hemiplegic migraine 1 mouse model after the induction of cortical spreading depression. The Journal of Headache and Pain 2014 15(Suppl 1):B5. 